\title{
El papel de la radiología diagnóstica y terapéutica en el campo de la salud pública
}

\author{
Cari Borrás ${ }^{1}$ coordinadora del número especial
}

La publicación periódica de la Organización Panamericana de la Salud (OPS) La salud en las Américas, en su edición de 2002, contiene reflexiones sobre los cambios demográficos, socioeconómicos y epidemiológicos que han tenido lugar en la Región de las Américas en los últimos años y documenta el aumento de la esperanza de vida en todos los países hasta su promedio actual (con la exclusión de Haití) de más de 70 años (1). Según la edición de 2006 de la publicación electrónica de la OPS Estadísticas de salud de las Américas (2), que presenta los datos de 37 países, la esperanza de vida al nacer para el quinquenio de 2005-2010 en ambos sexos variará entre 65,4 (en Guyana) y 80,7 años (en Canadá) y será de 73,3 años en promedio, con una desviación estándar de 5,17 años (excepto en Haití, donde se prevé que esta cifra será de 53,5 años). El envejecimiento progresivo de la población representa un gran reto para los ministerios de salud, ya que la prevalencia de las enfermedades crónicas, que afectan a la población a edades más avanzadas, va en aumento.

Según las estadísticas de 2006, las 10 principales causas de muerte en 31 países de la Región explican entre 43,1\% y 59,8\% de las defunciones registradas en esos países (2). Las enfermedades cerebrovasculares se encuentran entre las 10 principales causas de muerte en ambos sexos en los 31 países y la cardiopatía isquémica, en todos menos en Dominica, Haití y Honduras. En hombres, el cáncer de próstata es una de las 10 principales causas de muerte en 17 países. Asimismo, las enfermedades crónicas de las vías respiratorias inferiores, la insuficiencia cardíaca y

\footnotetext{
Dirección postal: 1501 Forty-fourth Street, NW, Washington, D.C. 20007, Estados Unidos de América. Correo electrónico: cariborras@starpower.net
}

sus complicaciones, y las cardiopatías mal definidas se encuentran entre las principales causas de muerte en 14 de los países analizados. En las mujeres, la influenza y la neumonía están entre las 10 principales causas de muerte en 30 países; la cardiopatía isquémica, en 28; y la insuficiencia cardíaca, en 25. Los cánceres de mama y útero continúan figurando entre las 10 principales causas de muerte en 16 países, seguidos del cáncer de colon en seis países y del cáncer de tráquea, bronquios y pulmón en cinco (2).

En este contexto, las aplicaciones radiológicas cobran una enorme importancia. El diagnóstico de las enfermedades cerebrovasculares, cardiopatías y neoplasias se facilita gracias a la radiología diagnóstica, mientras que algunos de los tratamientos de elección para estas afecciones se basan en la radiología intervencionista. Muchas de las radiaciones empleadas, tanto en la radiología convencional como en la tomografía computarizada, son de tipo ionizante; otras, como las empleadas para la ecografía y la resonancia magnética, no lo son. Mientras que algunas técnicas dependen del uso de radiaciones producidas por equipos de rayos $X$, otras se basan en la visualización de sustancias radiactivas que se le administran al paciente y que el organismo absorbe. Para la radioterapia se pueden usar equipos de radiación electromagnética externa, como los aceleradores lineales, o fuentes radiactivas, como las unidades de cobaltoterapia y las fuentes selladas de cesio-137 utilizadas en las aplicaciones ginecológicas.

El objetivo fundamental de todas estas técnicas radiológicas es prolongar la vida de los pacientes y disminuir la morbilidad. A medida que se extiende su uso, esta tecnología adquiere mayor complejidad. La realización eficiente y segura de los procedimientos de diagnóstico y 
tratamiento con fuentes de radiación exige que el personal que los lleva a cabo esté adecuadamente formado y entrenado. El médico clínico, el especialista en radiología, el radiooncólogo, el especialista en medicina nuclear, el físico médico, los tecnólogos y el personal de enfermería son miembros de ese gran elenco interdisciplinario que conforma el personal de salud de los servicios de radiología. Southon (3) analiza los beneficios de contar con redes de profesionales que puedan respaldar las políticas nacionales relacionadas con los servicios de salud — que abarcan los de radiología - y mejorar la calidad de las prestaciones.

En su función rectora y normativa, los ministerios de salud son los responsables de velar por y garantizar la calidad de los servicios de radiología. Una manera de llevar a cabo esta tarea es mediante programas de acreditación. En el artículo de Jiménez et al. (4) se examinan las características potenciales de tales programas en países en desarrollo, en particular en el contexto de los servicios de imaginología básicos, como los de radiografía, fluoroscopia, mamografía y ecografía, mediante la fusión de los aspectos físicos y clínicos.

Es un hecho reconocido que los procedimientos de radiología deben estar sujetos a programas de control de la calidad con un fuerte componente de seguridad radiológica. Indudablemente, tales programas mejoran la calidad de la imagen radiológica y disminuyen la dosis de radiación que reciben el paciente y los operarios. Pero ¿aumentan los diagnósticos certeros? Esta cuestión fue estudiada por un grupo de investigadores que, con el apoyo de la OPS, evaluaron la calidad de los servicios de radiodiagnóstico en cinco países de la Región de características sociales y avances tecnológicos muy diferentes: Argentina, Bolivia, Colombia, Cuba y México. Fleitas et al. (5) presentan los resultados de esa investigación, que demuestra la gran importancia de la formación y experiencia de los radiólogos y de los técnicos de radiología para lograr más diagnósticos certeros. Esta investigación abarcó, entre otros, servicios de mamografía, tanto de tamizaje como de diagnóstico.

Someter a una mamografía a todas las mujeres a partir de cierta edad, aunque estén asintomáticas, es una estrategia de salud pública para la detección temprana del cáncer de mama que ha suscitado grandes polémicas. En este número especial, Feig (6) demuestra que los beneficios de dicha estrategia superan a las limitaciones propias de la poca sensibilidad de la técnica y a los riesgos relacionados con las dosis de radiación recibidas. La mamografía sigue siendo hoy en día la única técnica reconocida para el tamizaje del cáncer de mama.
La imaginología puede ser diagnóstica o intervencionista. Las técnicas de diagnóstico permiten obtener información morfológica (estática) o información fisiológica (dinámica). Para todo ello se dispone de los recursos de la radiología convencional, la ecografía, la resonancia magnética y la medicina nuclear, siendo esta última la única que permite realizar estudios fisiológicos además de morfológicos, para lo cual depende de la introducción de radiofármacos en el organismo del paciente. Una de las nuevas tecnologías, la tomografía por emisión de positrones (PET), ha revolucionado no solo el conocimiento que se tiene acerca de las funciones cerebrales humanas, sino que además se está convirtiendo en una herramienta imprescindible para la localización y delimitación de neoplasias. No existen muchos equipos de PET en América Latina y el Caribe; el Brasil es uno de los países más avanzados en este campo, como nos explica Robilotta (7).

Se debe destacar que los avances tecnológicos no han ocurrido solamente en el campo del diagnóstico. A medida que la incidencia de cáncer aumenta en el mundo, se buscan nuevas formas de tratamiento. Junto con la cirugía y la quimioterapia, la radioterapia ofrece posibilidades de curación y paliación que prolongan la supervivencia y disminuyen la morbilidad. Castellanos (8) nos informa acerca de las necesidades y retos en el campo de la radioterapia en la actualidad.

Para curar un tumor con radiaciones hay que aplicar dosis muy grandes de radiación. Las nuevas técnicas de imaginología permiten visualizar el volumen del área que debe tratarse y circunscribir la radiación a los tejidos afectados por el tumor. Sin embargo, a pesar de los avances descritos por Castellanos, es imposible impedir que los tejidos sanos alrededor del tumor se vean expuestos a alguna radiación, aunque sea a dosis más bajas. Los efectos de la radiación en estos tejidos son analizados por Hendry et al. (9), quienes cuantifican los riesgos en función de la dosis.

En radioterapia es esencial que la dosis prescrita coincida con la dosis que recibe el paciente. El Organismo Internacional de Energía Atómica y la Organización Mundial de la Salud establecieron en 1969 un programa de dosimetría postal que permite verificar si las unidades de radioterapia de alta energía están calibradas adecuadamente. En la Región de las Américas, la OPS administra este programa. Iżewska et al. (10) presentan y examinan los resultados de las evaluaciones realizadas de 1969 a 2003 en los países de América Latina y el Caribe y muestran los grandes adelantos que se han logrado. 
Lamentablemente, los errores en las dosis que reciben los pacientes sometidos a radioterapia no se deben solamente a la mala calibración de las unidades de tratamiento. Borrás (11) describe un error de sobreexposición de 28 pacientes en Panamá debido a la utilización inapropiada del sistema computarizado de planificación de tratamientos. Asimismo, describe las medidas tomadas por la institución y la OPS para prevenir errores semejantes en el futuro.

¿Cuándo se considera que una irradiación es excesiva? Las dosis usadas para la radioterapia son 1000 veces mayores que las que se utilizan para el diagnóstico. ¿Son todas peligrosas? ¿Cómo se aseguran los ministerios de salud de que la población no reciba dosis dañinas? ¿Quién controla las fuentes de radiación? ¿Quién permite su entrada o fabricación en el país? Arias (12) nos informa acerca del desarrollo de los programas de protección radiológica y define el papel de las autoridades reguladoras en ese sentido.

Según Arias, entre los tejidos y órganos más sensibles a la radiación se encuentran los del embrión y el feto. El miedo irracional a las radiaciones lleva a muchas mujeres embarazadas que han pasado por un examen o tratamiento radiológico a preocuparse por los efectos de la radiación en la criatura en gestación y se plantean la necesidad de interrumpir el embarazo. Brent (13) comparte con nosotros algunos de estos casos y los resultados del asesoramiento proporcionado a las mujeres en situaciones semejantes.
La población no solo les teme a las radiaciones ionizantes, sino también a las que no son ionizantes y emanan de campos electromagnéticos, como los de los teléfonos celulares. Toda radiación, sea ionizante o no, se tiene que regir por normas y estándares. Skvarca et al. (14) explican los límites de exposición y los protocolos de medición para las radiaciones no ionizantes, tomando como base su utilización en la Argentina.

Para ilustrar los diferentes aspectos de la radiología diagnóstica y terapéutica, se podrían haber solicitado contribuciones sobre muchos otros temas. Este campo es cada vez más amplio. El objetivo de los artículos seleccionados para este número especial es dirigir la atención de los lectores de la Revista Panamericana de Salud Públical Pan American Journal of Public Health hacia un campo - quizá desconocido, pero fascinante- que abre enormes posibilidades, impensables muy pocos años atrás. Gracias a las tecnologías propias de este campo, los pacientes ya no tienen que someterse a largas hospitalizaciones y pueden gozar de una vida más larga y de mejor calidad.

La radiología genera esperanzas, permite tener ilusiones. La intención de los artículos e informes presentados en este número especial es llevar al lector a profundizar en este novedoso campo. Quiero agradecerles inmensamente a todos los autores a quienes les solicité contribuciones originales para este número su desprendimiento y generosidad. Me gustaría pensar que gracias a estas contribuciones y al esfuerzo de los editores, en algún lugar de las Américas alguna persona se salvará o tendrá una vida de mejor calidad.

\section{REFERENCIAS}

1. Organización Panamericana de la Salud. Salud en las Américas, 2002. Washington, D.C.: OPS; 2002. (Documento Oficial no. 587).

2. Organización Panamericana de la Salud. Estadísticas de salud en las Américas, 2006. Washington, D.C.: OPS; 2006. hallado en: http:/ /www. paho.org/Spanish/DD/AIS/hsa200 6. htm. Acceso el 9 de agosto de 2006.

3. Southon G. The role of professional networks in radiology services. Rev Panam Salud Publica. 2006;20(2/3): 99-103.

4. Jiménez $P$, Borrás $C$, Fleitas I. Accreditation of diagnostic imaging services in developing countries. Rev Panam Salud Publica. 2006; 20(2/3):104-12.

5. Fleitas I, Caspani CC, Borrás C, Plazas MC, Miranda AA, Brandan $\mathrm{ME}$, et al. La calidad de los servicios de radiología en cinco países lati- noamericanos. Rev Panam Salud Publica. 2006;20(2/3):113-24.

6. Feig SA. Screening mammography: a successful public health initiative. Rev Panam Salud Publica. 2006; 20(2/3):125-33.

7. Robilotta CC. A tomografia por emissão de pósitrons: uma nova modalidade na medicina nuclear brasileira. Rev Panam Salud Publica. 2006 20(2/3):134-42.

8. Castellanos ME. Las nuevas tecnologías: necesidades y retos en radioterapia en América Latina. Rev Panam Salud Publica. 2006;20(2/3): 143-50.

9. Hendry JH, Jeremić B, Zubizarreta EH. Normal tissue complications after radiation therapy. Rev Panam Salud Publica. 2006;20(2/3):151-60.

10. Iżewska J, Vatnitsky S, Shortt KR. Postal dose audits for radiotherapy centers in Latin America and the
Caribbean: trends in 1969-2003. Rev Panam Salud Publica. 2006;20(2/3): 161-72.

11. Borrás C. Overexposure of radiation therapy patients in Panama: problem recognition and follow-up measures. Rev Panam Salud Publica. 2006;20(2/3):173-87.

12. Arias CF. La regulación de la protección radiológica y la función de las autoridades de salud. Rev Panam Salud Publica. 2006;20(2/3):188-97.

13. Brent RL. Counseling patients exposed to ionizing radiation during pregnancy. Rev Panam Salud Publica. 2006;20(2/3):198-204.

14. Skvarca J, Aguirre A. Normas y estándares aplicables a los campos electromagnéticos de radiofrecuencias en América Latina: guía para los límites de exposición y los protocolos de medición. Rev Panam Salud Publica. 2006;20(2/3):205-12. 